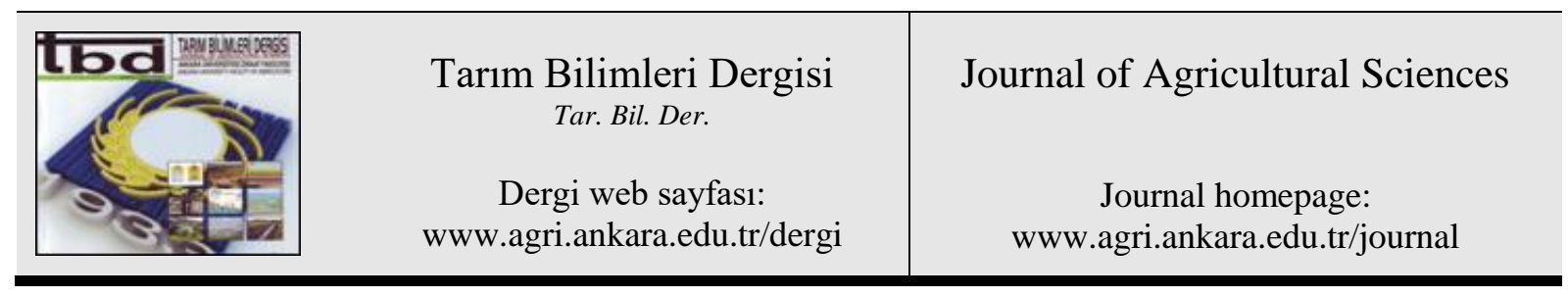

\title{
Mite Diversity (Acari) from Ornamental Plants in Erzurum in Turkey
}

\author{
Sultan ÇOBANOĞLU ${ }^{a}$, Kübra AKÇAKOYUNLUOĞLU ${ }^{b}$, Önder ÇALMAŞUR

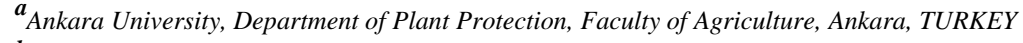

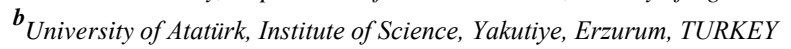

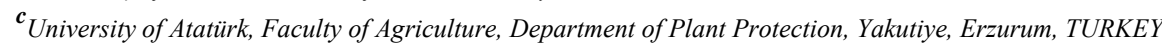

\author{
ARTICLE INFO \\ Research Article \\ Corresponding Author: Sultan ÇOBANOĞLU, E-mail: scobanoglu@ankara.edu.tr, Tel: +90 (312) 5961785 \\ Received: 26 January 2019, Received in Revised Form: 16 February 2019, Accepted: 27 February 2019
}

\section{AUTHORS ORCID ID}

(Sultan ÇOBANOĞLU: 0000-0002-3470-1548), (Kübra AKÇAKOYUNLUOĞLU: 0000-0002-5858-0779), (Önder ÇALMAŞUR: 0000-0001-8921-2381)

\begin{abstract}
Mite species belongs to Tetraychychidae (3); Tenupalpidae (2), Stigmaeidae (1), Tydeiidae (2), (Acari: Prostigmata); Phytoseiidae (10) (Acari: Mesostigmata) and Acaridae (1) (Astigmata) were identified on woody ornamental plants and shrubs in Erzurum (Eastern part of Turkey). The samples were collected from Erzurum (Centrum, Pasinler, Köprüköy, Horasan, Aziziye-Ilıca, Aşkale, Tortum, Uzundere and Çat districts) with a weekly interval between April to October during 2015 and 2016. Five species were phytophagous mites (belong to Tenuipalpidae and Tetranychidae), while the others are considered as predators or feed on microorganisms, neutral in their habitats. Nineteen mite species representing in three orders: Amblyseius andersoni (Chant), Kampimodromus aberrans (Oudemans), Euseius finlandicus (Oudemans), Typlodromus cotoneastri (Wainstein), Neoseiulus astutus (Beglyarov), Phytoseius finitimus Ribaga, Typhlodromus (Anthoseius)
\end{abstract}

kerkirae Swirski and Ragusa, Typhlodromus (Anthoseius) recki (Wainstein), Paraseiulus soleiger (Ribaga), Neoseiulella tiliarum (Oudemans), Zetzellia mali (Ewing), Tydeus kochi Oudemans, Tydeus californicus (Banks), Tetranychus urticae Koch, Bryobia rubrioculus (Scheuten), Bryobia praetiosa Koch, Cenopalpus pulcher (Canestini \& Fanzago) Brevipalpus californicus (Banks), Tyrophagus putrescentiae. These results showed that Erzurum has rich biodiversity especially concerning predatory mite fauna. T. urticae (Schrank), was the most abundant and common phytophagous species $(53.11 \%)$ while some other species were represented only one specimen (Neoseiulus astutus (Beglyarov) (Phytoseiidae). Most preferred hosts plants were Philadelphus coronarius L. (Hydrangeaceae) (8), Malus coronaria L. (Rosaceae) (7) and Rosa canina L. (Rosaceae) (6) while Syringa vulgaris L. (Oleaceae), Salix sp. (Salicaceae) and Rosa pisiformis (Christ) (Rosaceae) were populated by only (2) and (1) mite species respectively.

Keywords: Acari; Erzurum; Ornamental plants; Phytoseiidae; Tetranychidae

(C) Ankara Üniversitesi Ziraat Fakültesi

\section{Introduction}

Ecological condition in Erzurum and its neighbourhoods area are not suitable for several exotic outdoor ornamental plants to grow. However, the area is located phytogeographically in ancient Mesopotamian area. Therefore, plant species which can grow naturally in these areas can provide a rich ecological diversity. Erzurum is located at 2000 $\mathrm{m}$ altitude. Ornamental plants have several functional and aesthetic landscape values by being a native tree and small tree species of the region (Irmak 2013).

Several surveys were conducted to determine the mite species associated with woody ornamentals and shrubs of World-wide. It was reported several new mite species for the Hungary (Ripka et al 2002; 2005; Szabó et al 2009). In Turkey, some mites especially belong to Tetranychidae, Tenuipalpidae, Eriophyidae and Phytoseiidae 
species were identified on ornamental and park plants (Alaoğlu 1991; 1996; Faraji et al 2011; Çobanoğlu et al 2016).

There are limited numbers of the study exists on agricultural and park areas in Erzurum. Some plant-parasitic and phytoseiid species were reported in Erzurum and Erzincan previously. Bryobia rubrioculus Scheuten, Tetranychus urticae Koch, Typhlodromus kazachstanicus Wainstein (Ecevit 1981); Euseius finlandicus (Oudemans), Kampimodromus aberrans Oudemans, Paraseiulus soleiger (Ribaga), Paraseiulus talbii (AthiasHenriot), Phytoseius echinus Wainstein \& Arutunjan, Neoseiulella tiliarum (Oudemans) and Typhlodromus (Anthoseius) rhenanus (Oudemans) (Alaoğlu 1996). Beside this Neoseiulus zwoelferi (Dosse) and Proprioseiopsis okanagensis (Chant) species were reported in that region (Çobanoğlu 1989).

Therefore, it is a major necessitates a thorough investigation into the mites associated with ornamental plants for determination of the mite biodiversity in Erzurum. Survey studies can provide detection of predatory species are rich which are potential for biological control of economical important pests on ornamental plants.

The goal of the study is to determine mite species on shrubs and woody ornamental plants in Erzurum plateau during 2015-2016.

\section{Material and Methods}

The surveys were carried out on woody ornamentals and shrubs in Erzurum. The samples were collected 11 different host plants: 1. Rosa canina L. (Rosaceae), 2. Rosa dumalis Bechst. (Rosaceae), 3. Rosa pisiformis (Christ) (Rosaceae), 4. Ribes aureum Pursh. (Grossulariaceae), 5. Philadelphia coronarius L. (Hydrangeaceae), 6. Robinia pseudoacacia L. (Fabaceae), 7. Hippophae salicifolia Robert (Elaeagnaceae), 8. Syringa vulgaris L. (Oleaceae), 9. Malus coronaria L. (Rosaceae), 10. Malus floribunda L. (Rosaceae), 11. Salix sp. with 9 different districts of Erzurum (Centrum, Pasinler, Köprüköy, Horasan, Aziziye (Ilıca), Aşkale, Tortum, Uzundere, and Çat), which is located Eastern part of Turkey, during 2015 and 2016 (Figure 1; Table 1).

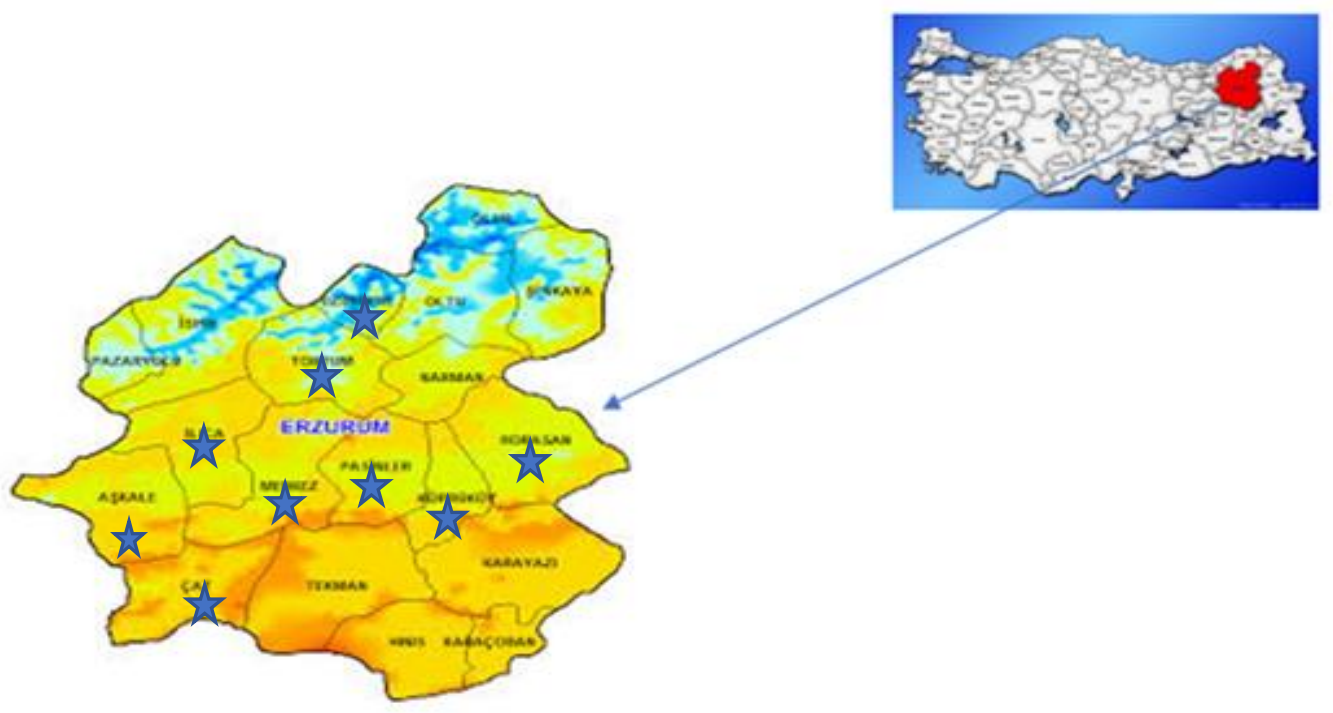

\section{Figure 1. Sampling localities: Erzurum (Eastern Part of Turkey) ( $九$ ( )}

The samples were deposited in the mite collection at Ankara University and Atatürk University Plant Protection Department of Turkey. The samples were collected from April to October of each year (2015 and 2016) with a weekly interval. The mites were collected randomly from the different height of the plants. The sampling was conducted from ornamental plants in landscape areas such as parks, gardens, roadsides, school and home gardens. 
All the mite samples were extracted by Berlese funnel. The mites were kept in $70 \%$ ethanol and afterwards, cleared in Lacto-phenol solution and prepared in Hoyer's medium, later they dried for $15-20$ days at $50{ }^{\circ} \mathrm{C}$ (Henderson 2001).

The collections were made by K. Akçakoyunluoğlu (Atatürk University).

All the identification of the samples were made by S. Çobanoğlu, according to; Jeppson et al (1975), Kolodochka (1978), Papadoulis et al (2009), Faraji et al (2011), Seeman \& Beard (2011) and Çobanoğlu et al (2016). World distribution and host range are considered, according to by Migeon \& Dorkeld (2006-2016); Moraes et al (2004) and Demite et al (2015). GPS data of the collection sites are shown in Table (1).

Table 1- Coordinates of the sampling localities

\begin{tabular}{ll}
\hline Location & \multicolumn{1}{c}{ GPS } \\
\hline Atatürk University Campus & $\mathrm{N}^{\circ} 9^{\circ} 53^{\prime} 59.1^{\prime \prime}, 041^{\circ} 14^{\prime} 19.0^{\prime \prime} \mathrm{E} 1880 \mathrm{~m}$ \\
Pasinler & $40^{\circ} 02^{\prime} 56.0^{\prime \prime} \mathrm{N}, 41^{\circ} 35^{\prime} 21.7^{\prime \prime} \mathrm{E} 1660 \mathrm{~m}$ \\
Uzundere & $40^{\circ} 32^{\prime} 52.7^{\prime \prime} \mathrm{N}, 41^{\circ} 34^{\prime} 23.6^{\prime \prime} \mathrm{E}, 1089 \mathrm{~m}$ \\
Çat & $39^{\circ} 35^{\prime} 42.4^{\prime \prime} \mathrm{N}, 40^{\circ} 57^{\prime} 59.6^{\prime \prime} \mathrm{E}, 1918 \mathrm{~m}$ \\
Aziziye & $39^{\circ} 55^{\prime} 09.3^{\prime \prime} \mathrm{N}, 41^{\circ} 12^{\prime} 20.7^{\prime \prime} \mathrm{E}, 1800 \mathrm{~m}$ \\
Aziziye-Dadaşkent & $39^{\circ} 55^{\prime} 09.3^{\prime \prime} \mathrm{N}, 041^{\circ} 12^{\prime} 20.7^{\prime \prime} \mathrm{E}, 1806 \mathrm{~m}$ \\
Tortum & $40^{\circ} 18^{\prime} 35^{\prime \prime} \mathrm{N}, 41^{\circ} 31^{\prime} 33^{\prime \prime} \mathrm{E}, 1637 \mathrm{~m}$ \\
Tortum Chelles area, & $40^{\circ} 33^{\prime} 55.6^{\prime \prime} \mathrm{N}, 41^{\circ} 35^{\prime} 46.2^{\prime \prime} \mathrm{E}, 1009 \mathrm{~m}$ \\
Aşkale & $39^{\circ} 56^{\prime} 03.1^{\prime \prime} \mathrm{N}, 40^{\circ} 43^{\prime} 32.8^{\prime \prime} \mathrm{E}, 1662 \mathrm{~m}$ \\
Aşkale-Çayköy & $39^{\circ} 56^{\prime} 44.2^{\prime \prime} \mathrm{N}, 040^{\circ} 48^{\prime} 18.5^{\prime} \mathrm{E}, 1720 \mathrm{~m}$ \\
Abdurrahman Gazi Forest & $39^{\circ} 52^{\prime} 36.0^{\prime \prime} \mathrm{N}, 41^{\circ} 18^{\prime} 35.22^{\prime \prime} \mathrm{E}, 2170 \mathrm{~m}$ \\
Erzurum centrum, & $39^{\circ} 48^{\prime} 59.7^{\prime \prime} \mathrm{N}, 41^{\circ} 04^{\prime} 32.8^{\prime \prime} \mathrm{E}, 1880 \mathrm{~m}$ \\
Palandöken & $39^{\circ} 48^{\prime} 41.2^{\prime \prime} \mathrm{N}, 041^{\circ} 07^{\prime} 10.0^{\prime \prime} \mathrm{E}, 1990 \mathrm{~m}$ \\
\hline
\end{tabular}

\section{Results and Discussion}

In total, 241 specimens were collected from shrubs and woody ornamental plants which were 98 from Rosa canina L. (Rosaceae), 67 from $R$. aureum and 39 from $P$. coronarius.

In a total of 19 mite species were identified in 3 different orders and 6 families. Three species of Tetranychidae, two Tenuipalpidae, ten Phytoseiidae and one species from Astigmata were identified. In these Tetranychidae and Tenuipalpidae species are plant-parasitic while one species of Acaridae is saprophagous and the rest are predatory species (Phytoseidae and Stigmaeidae) while Tydeidae includes neutral species (Table 2, 3).

Table 2- List of identified mite species in Erzurum-Turkey

\begin{tabular}{|c|c|c|}
\hline Order & Family & Mite species \\
\hline Mesostigmata & Phytoseiidae & $\begin{array}{l}\text { Amblyseius andersoni (Chant) } \\
\text { Kampimodromus aberrans (Oudemans) } \\
\text { Euseius finlandicus (Oudemans ) } \\
\text { Typlodromus cotoneastri (Wainstein) } \\
\text { Neoseiulus astutus (Beglyarov) } \\
\text { Phytoseius finitimus Ribaga } \\
\text { Typhlodromus (Anthoseius) kerkirae Swirski and Ragusa } \\
\text { Typhlodromus (Anthoseius) recki (Wainstein) } \\
\text { Paraseiulus soleiger (Ribaga) } \\
\text { Neoseiulella tiliarum (Oudemans) }\end{array}$ \\
\hline \multirow[t]{4}{*}{ Prostigmata } & Stigmaeidae & Zetzellia mali (Ewing) \\
\hline & Tydeidae & $\begin{array}{l}\text { Tydeus kochi Oudemans } \\
\text { Tydeus californicus (Banks) }\end{array}$ \\
\hline & Tetranychidae & $\begin{array}{l}\text { Tetranychus urticae Koch } \\
\text { Bryobia rubrioculus (Scheuten) } \\
\text { Bryobia praetiosa Koch }\end{array}$ \\
\hline & Teuipalpidae & $\begin{array}{l}\text { Cenopalpus pulcher (Canestini \& Fanzago) } \\
\text { Brevipalpus californicus (Banks) }\end{array}$ \\
\hline Astigmata & Acaridae & Tyrophagus putrescentiae (Schrank) \\
\hline
\end{tabular}




\subsection{Phytoseiidae berlese}

During surveys, 10 Phytoseiidae species were identified.

Amblyseius andersoni (Chant) 1957

Material examined - Pasinler, 11.VII.2015 (2へえへ) (Rosa dumalis subsp. bossieri). Uzundere, 12.VII.2015

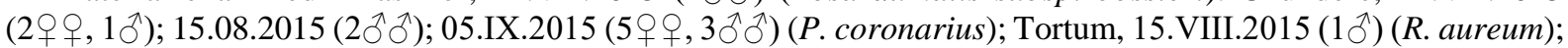
(1ㅇ) (R. dumalis).

Comments: Amblyseius andersoni is a common predatory mite species in Turkey; It was reported on Citrus spp. Convolvulus sp., Corylus, Fagus, Fragaria, Juglans, Lycopersicum, Malus, Populus, Prunus, Pyracantha, Rubus, Sambucus, Solanum and Tilia sp. from Ankara, Adana, Adapazarı, Antalya, Bartın, Bolu, Bursa, Edirne, Giresun, Hatay, Istanbul, Kırklareli, Rize, Sakarya, Tekirdağ, Tokat and Trabzon (Faraji et al 2011; Kumral \& Çobanoğlu 2015 ). A. andersoni was collected in Erzurum as 16 specimen 7.05\% (Table 3).

Table 3- Mite species and host plants range*

\begin{tabular}{|c|c|c|c|c|c|c|c|c|c|c|c|c|c|c|}
\hline \multirow{2}{*}{ Family } & \multirow{2}{*}{ Mite species } & \multicolumn{11}{|c|}{ Host plants } & \multirow{2}{*}{$\begin{array}{l}\text { Number of } \\
\text { specimens }\end{array}$} & \multirow{2}{*}{$\begin{array}{c}\text { Ratio } \\
(\%)\end{array}$} \\
\hline & & 1 & 2 & 3 & 4 & 5 & 6 & 7 & 8 & 9 & 10 & 11 & & \\
\hline \multirow{3}{*}{ Tetranychidae } & T. urticae & + & + & + & + & + & + & - & - & + & + & - & 128 & 53.11 \\
\hline & B. rubrioculus & - & - & - & - & + & - & - & - & + & - & - & 3 & 1.24 \\
\hline & B. praetiosa & - & + & - & - & - & - & - & - & - & - & - & 1 & 0.41 \\
\hline \multirow{9}{*}{ Phytoseiidae } & A. andersoni & - & + & - & + & + & - & - & - & - & - & - & 17 & 7.05 \\
\hline & N. astutus & - & - & - & - & - & - & + & - & - & - & - & 1 & 0.41 \\
\hline & T. cotoneastri & - & - & - & + & - & - & - & - & - & - & - & 1 & 0.41 \\
\hline & T. (A.) kerkirae & + & - & - & - & + & - & - & - & - & - & - & 5 & 2.07 \\
\hline & $T(A)$. recki & - & - & - & - & - & - & + & - & - & - & - & 1 & 0.41 \\
\hline & P. finitimus & - & - & - & + & - & - & - & - & + & - & - & 5 & 2.07 \\
\hline & E. finlandicus & + & + & - & + & + & + & - & + & + & - & - & 45 & 18.67 \\
\hline & K. aberrans & - & - & - & - & - & - & - & - & + & + & - & 9 & 3.73 \\
\hline & P. soleiger & - & - & - & - & - & - & - & - & - & - & + & 2 & 0.82 \\
\hline \multirow{3}{*}{ Tydeidae } & N. tiliarum & + & - & - & - & + & - & - & - & + & - & - & 4 & 1.65 \\
\hline & T. kochi & - & - & - & - & - & + & - & + & - & - & - & 2 & 0.82 \\
\hline & T. californicus & - & + & - & - & - & - & - & - & + & - & + & 11 & 4.56 \\
\hline \multirow{2}{*}{ Tenupalpidae } & B. californicus & - & - & - & - & + & - & - & - & - & - & - & 1 & 0.41 \\
\hline & C. pulcher & - & - & - & + & + & - & - & - & - & + & - & 3 & 1.25 \\
\hline Stigmaeidae & Z. mali & + & - & - & - & - & - & - & - & - & - & - & 1 & 0.41 \\
\hline Acaridae & T. putrescentia & + & - & - & - & - & - & - & - & - & - & - & 1 & 0.41 \\
\hline \multirow{2}{*}{\multicolumn{2}{|c|}{ In total }} & & & & & & & & & & & & 241 & 100.00 \\
\hline & & & & & & ur & & & & & & 7. $\mathrm{Hi}_{\mathrm{T}}$ & ophae salici & \\
\hline \multicolumn{2}{|c|}{ 2.Rosa dumalis } & \multicolumn{8}{|c|}{ 5.Philadelphia coronaria } & \multicolumn{5}{|c|}{ 8.Syringa vulgaris } \\
\hline \multicolumn{2}{|c|}{ 3.Rosa pisiformis } & \multicolumn{8}{|c|}{ 6.Robinia pseudoacacia } & \multicolumn{5}{|c|}{ 9-Malus coronaria } \\
\hline \multicolumn{2}{|c|}{ 10. Malus floribunda } & \multicolumn{8}{|c|}{ 11. Salix sp. } & & & & & \\
\hline
\end{tabular}

Euseius finlandicus (Oudemans 1915)

Material examined - Atatürk University, 02.VII.2015 (10 ふふ઼) (P. coronarius); Aşkale, 11.VI.2015 (3우, $5 \hat{\jmath})$ (R. dumalis); Pasinler, 11.VII.2015 (3ठ̋̋) (R. aureum), S. vulgaris L., M. hybrida; Uzundere, 05.IX.2015

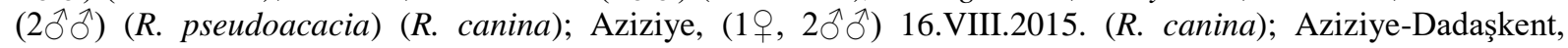


14.VIII.2015 (1ठ) (R. dumalis); Tortum Falls, 12.VII.2015 (5ㅇ, 8 §す) (M.communis); Tortum, 12.VII.2015 $(5 \hat{\jmath})($ R. aureum).

Comments: Euseius finlandicus is common on different plants throughout Turkey (Faraji et al 2011). It was recorded from eggplants, tomatoes, pepper, nightshade plants and Datura stramonium L. (Rosaceae) from Ankara, Bursa and Yalova Region of Turkey (Çobanoğlu \& Kumral 2014; 2016; Kumral \& Çobanoğlu 2016). It was identified from Erzurum (Alaoğlu 1996). It is abundant in Erzurum on parks and ornamental plant at 18.67\%.

Kampimodromus aberrans (Oudemans 1930)

Material examined - Uzundere, 07.VI.2015 (1 9 ) (M. cronoria); Tortum Falls, 07.VI.2015; 12.VIII.2015 (8우) (M. floribunda).

Comments: Kampimodromus aberrans is abundant on various plants, throughout Turkey. It was mentioned from different orchards, park plants, woody ornamentals and shrubs in Turkey (Alaoğlu 1996; Faraji et al 2011). It was collected in Erzurum at 3.73\% (Table 3).

Neoseiulus astutus (Beglyarov 1960)

Material examined - Aşkale-Çayköy, 05.IX.2015, (1ㅇ) (H. salicifolia)

Comments: Neoseiulus astutus was recorded in Ankara on Salix babylonica L. (Salicaceae) by Çobanoğlu (2002). It is a rare species and presented by only one specimen.

Paraseiulus soleiger (Ribaga1904)

Material examined - Aşkale-Çayköy, 06.VI.2016 (2 + ) (Salix sp.)

Comments: P. soleiger was found on M. communis, stone fruits; Prunus avium L., Prunus. persica L., Prunus domestica L. (Rosaceae), Ulmus sp. and Vitis vinifera L. (Vitaceae), from Adana, Ankara, Amasya, Erzincan, Erzurum, Gümüşhane, Isparta, Istanbul, Kastamonu, Manisa, Nevşehir, Niğde, Tekirdağ, Tokat and Van Lake Basin (Alaoğlu 1996; Faraji et al 2011). It was represented only two specimens.

Phytoseius finitimus Ribaga (1904)

Material examined - Aziziye- Dadaşkent, (2q⿱日)) (R. aureum) Tortum, 05.IX.2015 (2q); Tortum Falls, 05.IX.2015 (1우) (M. oronaria (L).

Comments: P. finitimus is a very common species throughout Turkey. This species was reported on Ailanthus sp., Citrus spp., Clematis vitalba, Cornus mas, Corylus avellana, Cydonia vulgaris, Ficus carica, Malus communis, Morus nigra, Prunus communis, Prunus domestica, Prunus spinosa, Rhamnus sp., Ribes sp., Rosa sp., Solanum melongena, Rubus sp., Ulmus campestris, Ulmus sp., Vitis vinifera (Çobanoğlu \& Kumral 2014; Faraji et al 2011). It is collected at $2.07 \%$ (Table 3).

Typlodromus cotoneastri Wainstein (1961)

Material examined - Tortum, 05.IX.2015, (1우) (R. aureum).

Comments: T. cotoneastri was reported on Betulaceae, Cornaceae, Rosaceae, Fagaceae, Moraceae, Pinaceaea, Ulmaceae, Caprifoliaceae and Vitaceae. It is distributed Ankara, Antalya, Bitlis, Edirne, Erzincan, Karabük, Kırklareli, Tekirdağ (Faraji et al 2011). It is collected in Erzurum at $0.41 \%$.

Typhlodromus (Anthoseius) kerkirae Swirski \& Ragusa (1976)

Material examined - Uzundere- Centrum, 05.IX.2015 (5 9 ) (R.canina). 
Comments: It was collected on Quercus sp in Adana (Döker et al 2016). It s collected at 2.07\% in Erzurum.

Typhlodromus (Anthoseius) recki Wainstein (1958).

Material examined - Aşkale-Çayköy, (1+) (H. salicifolia).

Comments: This species was collected from Citrus spp., Clematis vitalba L. (Ranunculaceae), M. communis, Pinus nigra J. F. Arnold, Pyrus elaeagnifolia Kotschyana (Rosaceae), Ribes sp., R. canina., V. vinifera in Adapazarı, Amasya, Ankara, , Burdur, Bursa, Edirne, Gümüşhane, İçel, Isparta, Istanbul, Izmir, Kars, Kastamonu, Konya, Muğla, Nevşehir, Niğde, Tekirdağ, Tokat, Zonguldak (Faraji et al 2011). It is collected as one specimen.

Neoseiulella tiliarum (Oudemans 1930).

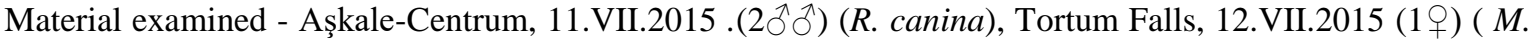
cronoria); Uzundere-centrum, 05.IX.2015 (1へ) (P. coronarius).

Comments: It was reported; C. avellana, Crataegus sp., Juglans regia L. (Juglandaceae), M. communis, Prunus cerasus L. (Rosaceae), P. domestica, from; Ankara, Amasya, Burdur, Bursa, Edirne, Erzincan, Erzurum, Gümüşhane, Isparta, Istanbul, Kastamonu, Konya, Nevşehir, Niğde, Tekirdağ, Tokat, Yalova (Alaoğlu 1996; Faraji et al 2011). It is collected at $1.65 \%$.

\subsection{Family tenuipalpidae berlese}

Cenopalpus pulcher Pritchard \& Baker (1958)

Material examined- Aziziye-Dadaşkent; 29.VI.2015 (1+) (R. aureum); 05.IX.2015 (1 +) (P. coronarius); Tortum falls, 12.VIII.2015 (1우) (M. floribunda).

Comments: Tenuipapids are plant parasitic species and known as Flat Mites. It was reported from Ankara, Bursa, Istanbul, Izmir, Konya Niğde, Samsun and Tokat from pome and stone fruits (Çobanoğlu et al 2016). It is presented at $1.25 \%$.

Brevipalpus californicus (Banks 1904)

Material examined - Uzundere, 05.IX.2015 (1ㅇ) (P. coronarius).

Comments: This species was collected on Citrus trees in Mersin and from Aegean vineyards (Göven et al 1999). B. californicus is rare species and collected as only one specimen.

\subsection{Family tetranychidae donnadieu}

\section{Tetranychus urticae Koch (1836)}

Material examined - Atatürk Univ. Campus, 27.V.2015 (1) (R. aureum), 05.VII.2015 (38 $Q$ ) $)(R$. canina). Pasinler, 06.VI.2015 (1), 11.VI.2015 (1+), 16.VIII.2015 (4우) (R. aureum), (1ㅇ) (M. hybrida);

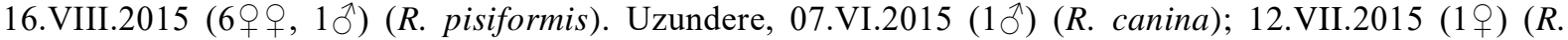
pseudoacacia); 12.VII.2015 (9우) (P. coronarius); 12.VII.2015 (1우시) (R. dumalis); 05.IX.2015 (2우) (R. canina), 05.IX.2015(9우) (P. coronarius); (1ㅇ) (R. pseudoacacia). Çat, 11.VII.2015 (3@, $8 \hat{\jmath})(R$. canina). Aziziye, 11.VI.2015 (6우) (R. aureum). Aziziye-Dadaşkent, 14.VIII.2015 (1 + ); 15.VIII.2015 (2우) (R. aureum), Tortum, 12.VII.2015 (1今) (R. canina); 12.VII.2015 (3우) (R. aureum); 15.VIII.2015 $(1+)(M$. communis $)$. 05.IX.2015 $(19)(R$. pisiformis $) ; 05 . I X .2015(3 \circ+)(R$. aureum).Tortum Falls area, 15.VIII.2015 (1ㅇ) (M. communis). Aşkale, 16.VIII.2015, (2q+q) (R. dumalis); 16.VIII.2015 (5우, $\left.1 \delta^{\Uparrow}\right)(R$. aureum). Abdurrahman Gazi Forest, 30.VIII.2015, (1 9$)$, (R. aureum); Erzurum centrum, 30.VIII.2015 (1ㅇ) (P. coronarius); (5우, $3 \hat{\jmath})$ (R. pisiformis); Palandöken, 30.VIII.2015 (2우) (R. dumalis). 
Comments: T. urticae is an important plant parasitic mite species which has distributed world-wide with more than 150 host plants and distributed all around Turkey (Ecevit 1981; Çobanoğlu \& Kumral 2014; Kumral \& Çobanoğlu 2015; 2016). It is very common on the ornamental plants in Erzurum. It was found $53.11 \%$ of the identified samples. It was collected from eight host plant species in Erzurum (Table 3).

Bryobia rubrioculus (Scheuten 1857)

Material examined - University Campus-Erzurum, 27.V.2015 (2우) (M. hybrida); Uzundere- Centrum, 05.IX.2015 (1ㅇ) (P. coronarius).

Comments: Bryobia is belongs of Bryobiinae and present several important species worldwide (Migeon \& Dorkeld 2006-2016). It is a serious pests on apple, apricot, plum, each and walnut trees. It was reported on Corylus avellana, citrus, Cotoneaster horizontalis Decne. (Rosaceae), Lonicera tatarica L. (Caprifoliaceae), Mahonia aquifolium (Pursh) (Berberidaceae), M. floribunda, Prunus cerasus, Thuja orientalis L. (Cupressaceae), V. vinifera from, Adana, Amasya, Ankara, Denizli, Çanakkale, Erzurum, Izmir, Manisa, Niğde Van and Black Sea region (Ecevit 1981; Göven et al 1999; Uysal et al 2001; Özman \& Çobanoğlu 2001). Bryobia species collected mostly on neglected trees. It was found (1.24\%) among the other mite species on P. coronoria and M. coronoria (Table 3$)$.

Bryobia praetiosa Koch (1836)

Material examined - Aziziye-Dadaşkent; 29.VI.2015, (1ㅇ) R. dumalis.

Comments: This species was reported Ankara on Fragaria ananassa, M.communis and Ficus carica (Uysal et al 2001). It was found $0.41 \%$ in Erzurum.

\subsection{Family stigmaeidae oudemans}

Zetzellia mali Ewing (1917)

Material examined - Aşkale-Centrum, 11.VII.2015 (1ㅇ) (Rosa canina)

Comments: Zetzellia mali is important predacious mite, it was reported from Ankara, Bilecik, Bursa, Samsun Van and Tokat (Doğan 2007; Çobanoğlu \& Kumral 2014; Kumral \& Çobanoğlu 2015). It is not very common in Erzurum and identified on $R$. canina as $0.41 \%$ (Table 3 )

\subsection{Family tydeidae kramer}

Tydeus californicus (Banks 1904)

Material examined - Aşkale-Çayköy, 06.VI.2015 (11 qq) (R. dumalis, M. cronoria, Salix sp.)

Comments: This is very small soft bodied mites and mostly feed on mites eggs. Tydeus californicus is a cosmopolitan species. It is also accepted as neutral species. T. californicus reported on hazelnut, pome and stone fruit trees all around Turkey (Çobanoğlu \& Kaźmierski 1999). It was represented by $4.56 \%$ in this region (Table 3)

Tydeus kochi Oudemans (1928)

Material examined-Pasinler, 11.VII.2015, (1+), (S.vulgaris); Uzundere, 05.IX.2015, (1 +) (R. pseudoacacia).

Comments: Tydeus kochi is a common species and reported on Fragaria ananassa Duchesne (Rosaceae) from Aydın (Çobanoğlu \& Kaźmierski 1999). We identified 0.82\% among the other mites (Table 3).

\subsection{Family acaridae}

Tyrophagus putrescentiae (Schrank 1781)

Material examined- Atatürk University Campus, 05.VII.2017 (1 \&) (R. canina) 
Comments: Tyrophagus putrescentiae prefer mostly stored products and common species throughout Turkey in different habitats and saprohagous mites. It was determined fresh onion fields in Izmir (Kılıç et al 2012), Sinapis arvensis L. (Brassicaceae) in Çanakkale (Kasap et al 2013), Lycopersicon esculentum, Solanum dulcamara L. and Solanum nigrum L. (Solanaceae) in Ankara, Bursa, Yalova (Çobanoğlu \& Kumral 2014; Kumral \& Çobanoğlu 2015). It represented by only one specimen.

\section{Conclusions}

Nineteen mite species are identified on woody ornamental plants in Erzurum. T. urticae, B. rubrioculus, T. kazachstanicus; E. finlandicus, K. aberrans, P. soleiger, P. talbii (Athias-Henriot), P. echinus, N. tiliarum, T. (A.) rhenanus, N. zwoelferi and P. okanagensis were identified previously (Ecevit 1981; Çobanoğlu 1989; Alaoğlu 1996). The rest of identified species in the parks and ornamental plants in Erzurum (A. andersoni, T. cotoneastri, N. astutus, P. finitimus, T. (A.) kerkirae, T. (A.) recki, Z. mali, T. kochi, T. californicus, B. praetiosa, C. pulcher, $B$. californicus and $T$. putrescentiae) are new records for this region. Erzurum has rich mite diversity especially concerning of beneficial mites because of rich host plants diversity and protected environment. Phytoseiidae members play important role as potential for biological control of economically important plant parasitic pests. Therefore, it is important to protect them in the environment. It will be useful to do more extensive studies in that area of Turkey.

\section{Acknowledgements}

This research was supported by the Atatürk University Scientific Research Project Coordination Unit (Project No: 2014/221). The authors are thankful to Prof. Dr. Yusuf KAYA (Atatürk University, Faculty of Science, Biology Department, Erzurum-Turkey) for determination of host plant samples. This study is cover by a part of master thesis of Kübra AKÇAKOYUNLUOĞLU.

\section{Reference}

Alaoğlu Ö (1991). Two new records of eriophyid mites (Acarina:Eriophyoidea) for the Turkish fauna. Atatürk Üniversitesi Ziraat Dergisi 22 (1): 72-77

Alaoğlu Ö (1996). Erzincan ve Erzurum illerinde Phytoseiidae (Acarina) faunası üzerinde çalışmalar. Selçuk Üniversitesi Ziraat Fakültesi Dergisi 9(11): 7-14

Banks N (1904). Four new species of injurious mites. Journal of the New York Entomological Society 12: 53-56

Beglyarov G A. (1960). Two new species of mite of the genus Typhlodromus Scheuten 1857 (Parasitiformes, Phytoseiidae). Entomologicheskoe Obozrenie, Russia 39: 956-958

Chant D A (1957). Descriptions of some Phytoseiidae mites (Acarina: Phytoseiidae). Part I. Nine new species from British Columbia with keys to the species of British Columbia. Part II: Redescripyion of eight species described by Berlese. The Canadian Entomologist 89(7): 289-308

Çobanoğlu S (1989). Türkiye için üç yeni faydalı akar (Acari, Phytoseiidae) türü. Türkiye Entomoloji Dergisi 13(4): 229-238

Çobanoğlu S (2002). Amblyseius astutus (Beglarov. 1960) (Acarina: Phytoseiidae), Türkiye faydalı akar faunası için yeni bir kayit. Türkiye Entomoloji Dergisi 26(3): 115-120

Çobanoğlu S \& Kaźmierski A (1999). Tydeidae and Stigmaeidae (Acari Prostigmata) from orchards, trees and shrubs in Turkey. Biological Bulletin of Poznan 36: 71-82

Çobanoğlu S \& Kumral N A (2014). Ankara, Bursa ve Yalova 1llerinde domates yetiştirilen alanlarda zararlı ve faydalı akar (Acari) biyolojik çeşitliliği ve popülasyon dalgalanması. Turkish Journal Of Entomology 38(2): 197-214

Çobanoğlu S, Ueckermann E A \& Sağlam H S (2016). The Tenuipalpidae of Turkey, with a key to species (Acari: Trombidiformes) Zootaxa. 4097(2): 151-186

Demite P R, Moraes G J de, McMurtry J A, Denmark H A. \& Castilho R C (2015). Phytoseiidae Database. Retrieved in December, 02, 2018 from www.lea.esalq.usp.br/phytoseiidae

Journal of Agricultural Sciences (Tarım Bilimleri Dergisi) 26 (2020) 236-245 
Doğan S (2007). Checklist of raphignathoid mites (Acari: Raphignathoidea) of Turkey. Zootaxa 1454: 1-26

Döker İ, Kazak C \& Karut K (2016). Contributions to the Phytoseiidae (Acari: Mesostigmata) fauna of Turkey: morphological variations, twelve new records, re-description of some species and a revised key to the Turkish species. Systematic and Applied Acarology 21 (4): 505-527. doi:http://dx.doi.org/10.11158/saa.21.4.10

Ecevit O (1981). Erzurum Elma Ağaçlarında Zararlı Bryobia rubrioculus Scheuten, Tetranychus urticae Koch., Aculus schlectendali (Nal.), Calepitrimerus haileyi Keifer (Acarina: Tetranychidae, Erophyiidae) ile Predatör Amblydromella kazachstanicus Wainstein (Acarina: Phytoseiidae) ve Populasyon İlişkileri. Ondokuzmayıs Üniversitesi Ziraat Fakültesi Yayınları Samsun, pp. 52

Ewing, H E ( 1917). New Acarina. Part II.- Description of new species and varieties from Iowa, Missouri, Illinois, Indiana, and Ohio. Bulletin American Museum of Natural History 38: 149-172

Faraji F, Çobanoğlu S \& Çakmak İ (2011). A checklist and a key for the Phytoseiidae species of Turkey with two new species records (Acari: Mesostigmata). International Journal of Acarology 37: 221-243

Göven M A, Çobanoğlu S, Güven B \& Topuz M (1999). "Investigations on the fauna of phytoseiid mites on vineyards in Aegean Region, 491-500". Proceedings of the Fourth Turkish National Congress of Biological Control, 26-29 Jan, Adana, Entomol Society Publication 9

Henderson R C (2001). Technique for positional slide-mounting of Acari. Systematic and Applied Acarology Special Publications 7: 1-4

Irmak M A (2013). Use of native woody plants in urban landscapes. Journal of Food, Agriculture and Environment 11(2): 1305-1309

Jeppson L R, Keifer H H \& Baker E W (1975). Mites Injurious to Economic Plants. University of California Press, Berkely, pp. 646

Kasap İ, Çobanoğlu S \& Pehlivan S (2013). Çanakkale ve Balıkesir illeri yumuşak çekirdekli meyve ağaçları ve yabancı otlar üzerinde bulunan predatör akar türleri. Türkiye Biyolojik Mücadele Dergisi 4(2): 109-124

Kılıç T, Çobanoğlu S, Yoldaş Z \& Madanlar N (2012). Izmir ilinde taze soğan tarlalarında bulunan akar (Acari) türleri. Turkish Journal Of Entomology 36(3): 401-411

Koch C L (1836). Deutsche Crustacea, Myriapoda, Arachnida. fasc. 1

Kolodochka L A (1978). Manual for the identification of plant-inhabiting phytoseiid mites. Akademiya Nauk SSSR, Zoological Institute. Naukova Dumka Kiev, pp. 78

Kumral N A \& Çobanoğlu S (2015). The potential of the nightshade plants (Solanaceae) as reservoir plants for pest and predatory mites. Turkish Journal Of Entomology 39(1): 91-108

Kumral N A \& Çobanoğlu S (2016). The Mite (Acari) biodiversity and population fluctuation of predominat species in eggplant. Journal of Agricultural Sciences 22: 261-274

Migeon A \& Dorkeld D (2006-2016). Spider Mites Web: A Comprehensive Database for the Tetranychidae. Montpellier, France: INRA. Retrieved in December, 02, 2018 from http://www.montpellier.inra.fr/CBGP/spmweb

Moraes G J, McMurtry J A, Denmark H A \& Campos C B (2004). A revised catalog of the mite family Phytoseiidae. Zootaxa 434: $1-494$

Oudemans A C (1915). Acarologische Aanteekeningen. LVI. Entomologische Berichten 4: 180-188

Oudemans A C (1928). Acarologische aanteekeningen XCIV. Entomologische Berichten 7: 374-382

Oudemans A C (1930). Acarologische Aanteekeningen. CI. Entomologische Berichten 8: 48-53

Özman S K \& Çobanoğlu S (2001). Current status of hazelnut mites in Turkey. Acta Horticulturae 556: 479-487

Papadoulis G Th, Emmanouel N G \& Kapaxidi E V (2009). Phytoseiidae of Greece and Cyprus (Acari: Mesostigmata). Indira Publishing House pp. 200 
Pritchard A E \& Baker E W (1958). The False Spider Mites (Acarina: Tenuipalpidae). University of California Publications in Entomology 14(3): 175-274

Ribaga C (1904). Gamasidi planticoli. Revista di patologia vegetale 10: 175-178

Ripka G, Fain A, Kaźmierski A, Kreiter S \& Magowski W Ł (2002). Recent data to the knowledge of the arboreal mite fauna in Hungary (Acari: Mesostigmata, Prostigmata, and Astigmata). Acarologia 42: 271-281

Ripka G, Fain A, Kazmierski A, Kreiter S \& Magowski W Ł (2005). New data to the knowledge of the mite fauna of Hungary (Acari: Mesostigmata, Prostigmata, and Astigmata). Acta Phytopathologica et Entomologica Hungarica 40: 159-176

Scheuten A (1857). Einiges über Milben. Archiv für Naturgeschichte 23(1): 104-114

Schrank, F P (1781). Enumeratio Insectorum Austriae Indigenorum. August Vindelicor, Klett, pp. 548

Seeman O D \& Beard J J (2011). Identification of exotic pest and Australian native and naturalised species of Tetranychus (Acari: Tetranychidae): Zootaxa 2961: 1-72

Swirski E \& Ragusa S (1976). Notes on predacious mites of Greece, with a description of Pve new species (Mesostigmata: Phytoseiidae). Phytoparasitica 4: 101-122

Szabó Á, Kóródi I \& Pénzes B (2009). Ragadozó atkák elôfordulása a Tokaj-hegyaljai borvidéken. (Predatory mite in Tokaj wine region). Növényvédelem 45: 21-27

Wainstein B A (1958). New species of mites of the genus Typhlodromus (Parasitiformes: Phytoseiidae) from Georgia [in Russian]. Soobshcheniya Akademii Nauk Gruzinskoy SSR 21(2): 201-207

Wainstein B A (1961). New species of mites of the genus Typhlodromus (Parasitiformes: Phytoseiidae) in Georgia. [in Russian]. Trudy Instituta Zoologii Akademii Nauk Gruzinskoy SSR 18: 153-162

Uysal C, Çobanoğlu S \& Ökten M E (2001). Ankara parklarında zarar yapan Tetranychoidea (Acarina: Prostigmata) türleri ve konukçularının saptanması üzerinde araştırmalar. Türkiye Entomoloji Dergisi 25(2): 147-160 